\title{
Shilajit: A Natural Phytocomplex with Potential Procognitive Activity
}

\author{
Carlos Carrasco-Gallardo, Leonardo Guzmán, and Ricardo B. Maccioni \\ Laboratory of Cellular and Molecular Neurosciences, International Center for Biomedicine (ICC) and University of Chile, \\ Millennium Building, Las Encinas 3370, Nuñoa, 780023 Santiago, Chile \\ Correspondence should be addressed to Ricardo B. Maccioni, rmaccion@manquehue.net
}

Received 22 August 2011; Accepted 17 December 2011

Academic Editor: Yoram Barak

Copyright ( $) 2012$ Carlos Carrasco-Gallardo et al. This is an open access article distributed under the Creative Commons Attribution License, which permits unrestricted use, distribution, and reproduction in any medium, provided the original work is properly cited.

\begin{abstract}
Shilajit is a natural substance found mainly in the Himalayas, formed for centuries by the gradual decomposition of certain plants by the action of microorganisms. It is a potent and very safe dietary supplement, restoring the energetic balance and potentially able to prevent several diseases. Recent investigations point to an interesting medical application toward the control of cognitive disorders associated with aging, and cognitive stimulation. Thus, fulvic acid, the main active principle, blocks tau self-aggregation, opening an avenue toward the study of Alzheimer's therapy. In essence, this is a nutraceutical product of demonstrated benefits for human health. Considering the expected impact of shilajit usage in the medical field, especially in the neurological sciences, more investigations at the basic biological level as well as clinical trials are necessary, in order to understand how organic molecules of shilajit and particularly fulvic acid, one of the active principles, and oligoelements act at both the molecular and cellular levels and in the whole organism.
\end{abstract}

\section{Introduction}

Shilajit also known in the north of India as salajit, shilajatu, mimie, or mummiyo is a blackish-brown powder or an exudate from high mountain rocks, especially in the Himalayans mountains between India and Nepal, although it has been also found in Russia, Tibet, Afghanistan, and now in the north of Chile, named as Andean Shilajit [1]. Shilajit has been known and used for centuries by the Ayurvedic medicine, as a rejuvenator and as antiaging compound. There are two important characteristics of a rasayana compound in the ancient Indian Ayurvedic medicine: that is, to increase physical strength and to promote human health [2]. The health benefits of shilajit have been shown to differ from region to region, depending on the place from which it was extracted $[3,4]$.

\section{Origins of Shilajit}

Considering its unique composition as a phytocomplex, very rich in fulvic acid, researchers hypothesize that Shilajit is produced by the decomposition of plant material from species such as Euphorbia royleana and Trifolium repens $[4,5]$. This decomposition seems to occur through centuries, and on this basis, shilajit is considered a millenary product of nature. However, further studies have identified that several other plant organisms may generate shilajit, such as molds as Barbula, Fissidens, Minium, and Thuidium and other species like Asterella, Dumortiera, Marchantia, Pellia, Plagiochasma, and Stephenrencella-Anthoceros [4].

\section{Molecular Composition of Shilajit}

Shilajit is composed mainly of humic substances, including fulvic acid, that account for around $60 \%$ to $80 \%$ of the total nutraceutical compound plus some oligoelements including selenium of antiaging properties $[6,7]$ (Figure 1). The humic substances are the results of degradation of organic matter, mainly vegetal substances, which is the result of the action of many microorganisms. Components are divided operationally in humins, humic acid, and fulvic acids according to their solubility in water at different $\mathrm{pH}$ levels. 


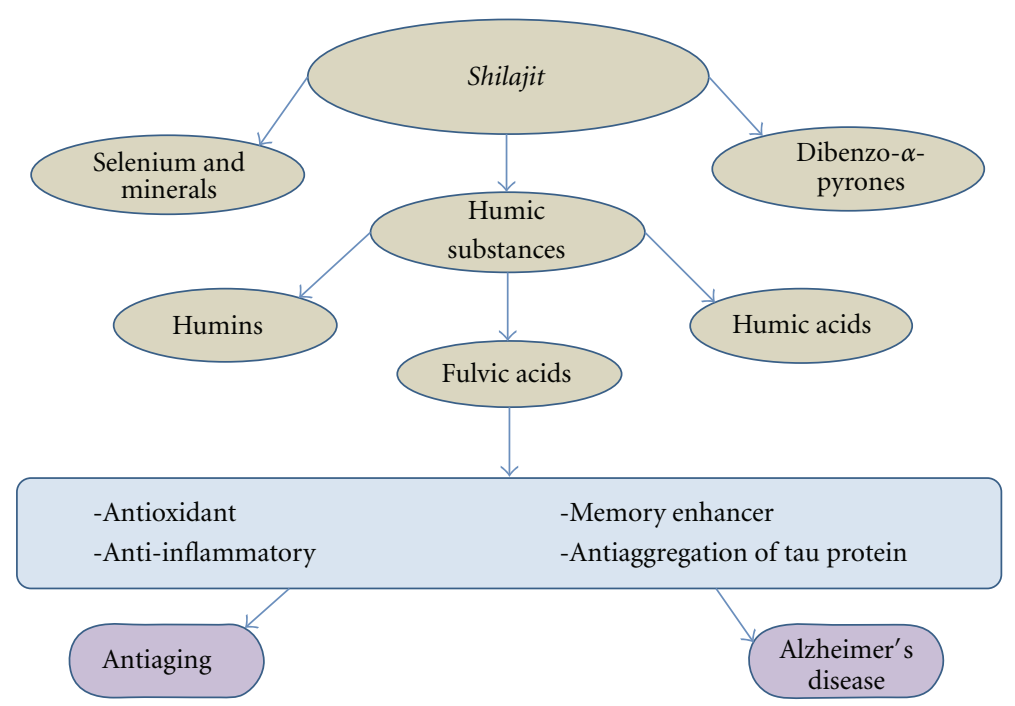

FIGURE 1: Shilajit, its main components, and potential uses based on properties of fulvic acid. This phytocomplex known as shilajit is mainly composed of humic substances. One of them, fulvic acid, is known by its properties such as antioxidant, anti-inflammatory, and memory enhancer. Novel investigations indicate that fulvic acid is an antiaggregation factor of tau protein in vitro [1], which projects fulvic acid as a potential anti-Alzheimer's disease molecule.

Humins are not soluble in water under any $\mathrm{pH}$ condition. Humic acid is soluble in water under alkaline conditions and has a molecular weight of 5-10 kDa. Fulvic acid is soluble in water under different $\mathrm{pH}$ conditions, and because of its low molecular weight (around $2 \mathrm{kDa}$ ), it is well absorbed in the intestinal tract and eliminated within hours from the body $[8,9]$. It is likely that the curative properties attributable to shilajit are provided by the significant levels of fulvic acids that shilajit contains, considering that fulvic acid is known by its strong antioxidant actions [9] and likely has systemic effects as complement activator [10]. Recent studies on the composition of Andean Shilajit in Chile have evidenced an ORAC index between 50 and 500 Trolox units/g of material, which is substantially higher than Noni and blueberries (Quinteros et al., unpublished data). In this context, shilajit seems to be a powerful antioxidant phytocomplex.

Other molecules present in shilajit preparations are eldagic acid, some fatty acids, resins, latex, gums, albumins, triterpenes, sterols, aromatic carboxylic acids, 3,4-benzocoumarins, amino acids, polyphenols, and phenolic lipids $[3,6$, 11]. Certainly its molecular composition varies from region to region. Newer investigations based on high-performance size exclusion chromatography (HP-SEC) show that shilajit contains specific molecular species of polysaccharides and lignins [10]. As humic components, humins, humic acids, and fulvic acids are found in all shilajit preparations, being the last one, fulvic acids, the biologically active compound, along with dibenzo- $\alpha$-pyrones, which acts as carrier of other substances [3].

\section{Traditional Uses of Shilajit}

Shilajit is an important, known component of the ayurvedic medicine given its characteristics as a rasayana. In this context, health benefits such as an increase in longevity,
TABLE 1: Morphometric study of primary cultured rat hippocampal cells exposed to Shilajit and the Brain Up-10 formulae that contain Shilajit plus complex B vitamins (Vit B6, B9, and B12).

\begin{tabular}{lccc}
\hline & Control & Shilajit** & $\begin{array}{c}\text { Brain } \\
\text { Up-10* }\end{array}$ \\
\hline $\begin{array}{l}\text { Neuronal cells per field } \\
\text { Percentage of cells with }\end{array}$ & $367 \pm 23$ & $345 \pm 42$ & $396 \pm 16.0$ \\
$\begin{array}{l}\text { neuronal processes } \\
\begin{array}{l}\text { Fraction of axon-like } \\
\text { processes }\end{array}\end{array}$ & 0.22 & 0.29 & 0.41 \\
Processes length $(\mu \mathrm{m})$ & $17.4 \pm 7.2$ & $26.0 \pm 4.5^{* *}$ & $39.6 \pm 8.0^{* *}$
\end{tabular}

Hippocampal cells were grown in Petri dishes in the presence of either $10 \mathrm{mg} / \mathrm{mL}$ Shilajit or the formulation of Brain Up-10 [30] plus vitamins of the B complex. In the control, cells were grown in culture medium without Shilajit or the formulation. Mean of 5 determinations $(n=5)$ (significance of differences with respect to control, $\left.{ }^{* *} P<0.001\right)$.

rejuvenating, and arresting aging roles have been attributed to it [3]. Traditionally, shilajit is consumed by people from Nepal and the North of India, and children usually take it with milk in their breakfast. The Sherpas claim to have shilajit as part of their diet; they constitute a population of strong men with very high levels of a healthy longevity. Our laboratory has found evidence on the high activity of the Andean form of shilajit in improving cognitive disorders and as a stimulant of cognitive activity in humans [1] (Table 1).

Considering the actions of fulvic acid in preventing tau self-aggregation into pathological filaments, this compound appears to be of interest for prevention of Alzheimer's disease [1]. Other common traditional uses include its action in genitourinary disorders, jaundice, digestive disorders, enlarged spleen, epilepsy, nervous disorders, chronic bronchitis, and anemia [2]. Shilajit has been also useful for the treatment of kidney stones, edema, and hemorrhoids, as an internal antiseptic, and to reduce anorexia. Also, it has been claimed 
in India to be used as yogavaha $[12,13]$, that is, as synergistic enhancer of other drugs. Organic components of shilajit play also a role in transporting different mineral substances to their cellular targets.

\section{Novel Investigations}

Preclinical investigations about shilajit indicate its great potential uses in certain diseases, and various properties have been ascribed, including (1) antiulcerogenic properties [14]; (2) antioxidant properties $[15,16]$; (3) cognitive and memory enhancer $[1,10,17]$; (4) antidiabetic properties [18]; (5) anxiolytic [12]; (6) antiallergic properties and immunomodulator $[2,19,20]$; (7) anti-inflammatory [21]; (8) analgesic [16]; antifungal properties [22]; (9) ability to interact positively with other drugs [23]; (10) protective properties in high altitudes [24]; (11) neuroprotective agent against cognitive disorders [1, and Farias et al. unpublished clinical trials]. Unfortunately shilajit lacks systematic documentation and well-established clinical trials on its antioxidative and immunomodulatory actions in humans, and it is expected that considering the reported benefits evidenced from trials will be obtained in the near future [25].

\section{Patenting}

A few patents already exist that protect the use of shilajit in India and Nepal, such as US Patent 5,405,613-vitamin/ mineral composition [26]; US Patent application number 20030198695-Herbo-mineral composition [27]; US Patent number 6,440,436-Process for preparing purified shilajit composition from native shilajit [28]; US Patent number 6,558,712 - Delivery system for pharmaceutical, nutritional and cosmetic ingredients [29]. Other recent patent about a phytocomplex with vitamins added is WO 2011/041920 [30].

\section{Potential Risks}

Studies indicate the shilajit consumption without preliminary purification may lead to risks of intoxication given the presence of mycotoxin, heavy metal ions, polymeric quinones (oxidant agents), and free radicals, among others [3]. Therefore, a purified, ready-for-use preparation for human consumption must be used. However, recent studies indicate that several ayurvedic products including shilajit and other Indian manufactured products commercialized by the Internet may contain detectable heavy metals levels as lead, mercury, and arsenic [31]. This study showed the presence of heavy metals and other minerals, including gems, is associated with the belief that when mixed with shilajit or other herbal preparations they generate a better response from the body in a synergic manner. This is what is known as rasa-shastra in ayurvedic medicine. Rasashastra experts claim that if this is prepared, administered, and consumed properly, it is safe and has therapeutic advantages [31]. It is worth considering that recent clinical reports indicate cases of lead poisoning in patients who have used ayurvedic products against weakening $[32,33]$.

\section{Commentary and Discussion}

Shilajit has a comfortable position as the rasayana because of its excellence, well known in the Eastern culture, and now being introduced with great interest in the occidental world. The vast majority of published papers on this theme are from India, leaving this sector of the planet as an expert in their field, since this is a product that is extracted, marketed, and investigated in these latitudes. However, this generates a segmentation of shilajit, relegating it only to what has always been assumed : a natural product that is part of natural alternative medicine and not as a result of medical and biotechnology innovation worldwide. This is evidenced quite clearly by reviewing the literature today, and note that the journals where studies on shilajit are published (jobs are plentiful) are mainly reviewed in the Eastern. Given this, it is necessary that shilajit break the cultural paradigm and enter into the rest of the world by the hand of rigorous research at the molecular and cellular levels, which could elucidate the interactions of the active ingredients of the different shilajit preparations with biomolecules. This will facilitate our understanding of their mechanisms of action.

\section{Conclusion}

Shilajit is a potent and very safe dietary supplement, potentially able to prevent several diseases, but its main medical application now appears to come from its actions in benefit of cognition and potentially as a dietary supplement to prevent Alzheimer's disease. In essence, this is a nutraceutical product. Considering the expected impact of shilajit applications in the medical field, especially in neurological sciences, more investigations at the basic biological level are necessary, and certainly well-developed clinical trials, in order to understand how its active principles act at molecular and cellular levels.

\section{Acknowledgments}

These investigations have been supported by a CORFO Project 10ANT 8051, VRI FONDEF project, and FONDECYT 1110373 from CONICYT and a grant from the Alzheimer's Association, USA. Authors acknowledge important collaboration of Constanza Maccioni.

\section{References}

[1] A. Cornejo, J. M. Jiménez, L. Caballero, F. Melo, and R. B. Maccioni, "Fulvic acid inhibits aggregation and promotes disassembly of tau fibrils associated with alzheimer's disease," Journal of Alzheimer's Disease, vol. 27, no. 1, pp. 143-153, 2011.

[2] S. Ghosal, "Chemistry of shilajit, an immunomodulatory Ayurvedic rasayan," Pure and Applied Chemistry, vol. 62, no. 7, pp. 1285-1288, 1990.

[3] R. N. Chopra, I. C. Chopra, K. L. Handa, and K. D. Kapoor, In Indigenous Drugs of India, U.N. Dhar \& Sons, Calcutta, India, 1958.

[4] S. P. Agarwal, R. Khanna, R. Karmarkar, M. K. Anwer, and R. K. Khar, "Shilajit: a review," Phytotherapy Research, vol. 21, no. 5, pp. 401-405, 2007. 
[5] S. Ghosal, J. P. Reddy, and V. K. Lal, "Shilajit I: chemical constituents," Journal of Pharmaceutical Sciences, vol. 65, no. 5, pp. 772-773, 1976.

[6] R. Khanna, M. Witt, M. Khalid Anwer, S. P. Agarwal, and B. P. Koch, "Spectroscopic characterization of fulvic acids extracted from the rock exudate shilajit," Organic Geochemistry, vol. 39, no. 12, pp. 1719-1724, 2008.

[7] P. Mittal, D. Kaushik, V. Gupta, P. Bansal, and S. Khokra, "Therapeutic potencial of "Shilajit Rasayana"-A Review," International Journal of Pharmaceutical and Clinical Research, vol. 1, no. 2, pp. 47-49, 2009.

[8] K. M. S. Islam, A. Schumacher, and J. M. Gropp, "Humic acid substances in animal agriculture," Pakistan Journal of Nutrition, vol. 4, pp. 126-134, 2005.

[9] A. V. Vucskits, I. Hullár, A. Bersényi, E. Andrásofszky, M. Kulcsár, and J. Szabó, "Effect of fulvic and humic acids on performance, immune response and thyroid function in rats," Journal of Animal Physiology and Animal Nutrition, vol. 94, no. 6, pp. 721-728, 2010.

[10] I. A. Schepetkin, G. Xie, M. A. Jutila, and M. T. Quinn, "Complement-fixing activity of fulvic acid from shilajit and other natural sources," Phytotherapy Research, vol. 23, no. 3, pp. 373-384, 2009.

[11] Y. C. Kong, P. P. H. But, K. H. Ng et al., "Chemical studies on a Nepalese Panacea-shilajit (I)," International Journal of Crude Drug Research, vol. 25, no. 3, pp. 179-182, 1987.

[12] S. Ghosal, J. Lal, S. K. Singh, R. K. Goel, A. K. Jaiwal, and S. K. Bhattacharya, "The need for formulation of shilajit by its isolated active constituents," Phytotherapy Research, vol. 5, no. 5, pp. 211-216, 1991.

[13] S. Ghosal, B. Mukherjee, and S. K. Bhattacharya, "Shilajita comparative study of the ancient and the modern scientific findings," Indian Journal of Indigenous Medicine, vol. 17, pp. 1-10, 1995.

[14] S. Ghosal, S. K. Singh, Y. Kumar et al., "Shilajit. 3. Antiulcerogenic of fulvic acids and 4-methoxy-6-carbomethoxybiphenyl isolated from shilaji," Phytotherapy Research, vol. 2, no. 4, pp. 187-191, 1988.

[15] S. Ghosal, S. Lata, Y. Kumar, B. Gaur, and N. Misra, "Interaction of shilajit with biogenic free radicals," Indian Journal of Chemistry B, vol. 34, pp. 596-602, 1995.

[16] S. K. Bhattacharya and A. P. Sen, "Effects of shilajit on biogenic free radicals," Phytotherapy Research, vol. 9, no. 1, pp. 56-59, 1995.

[17] A. K. Jaiswal and S. K. Bhattacharya, "Effects of shilajit on memory, anxiety and brain monoamines in rats," Indian Journal of Pharmacology, vol. 24, pp. 12-17, 1992.

[18] S. K. Bhattacharya, "Shilajit attenuates streptozotocin induced diabetes mellitus and decrease in pancreatic islet superoxide dismutase activity in rats," Phytotherapy Research, vol. 9, no. 1, pp. 41-44, 1995.

[19] C. Wang, Z. Wang, A. Peng, J. Hou, and W. Xin, "Interaction between fulvic acids of different origins and active oxygen radicals," Science in China, Series C, vol. 39, no. 3, pp. 267-275, 1996.

[20] S. Ghosal, J. Lal, S. K. Singh et al., "Mast cell protecting effects of shilajit and its constituents," Phytotherapy Research, vol. 3, no. 6, pp. 249-252, 1989.

[21] S. B. Acharya, M. H. Frotan, R. K. Goel, S. K. Tripathi, and P. K. Das, "Pharmacological actions of shilajit," Indian Journal of Experimental Biology, vol. 26, no. 10, pp. 775-777, 1988.

[22] Shalini and R. Srivastava, "Antifungal activity screening and hplc analysis of crude extract from Tectona grandis, shilajit,
Valeriana wallachi," Electronic Journal of Environmental, Agricultural and Food Chemistry, vol. 8, no. 4, pp. 218-229, 2009.

[23] M. A. Mirza, S. P. Agarwal, M. A. Rahman et al., "Role of humic acid on oral drug delivery of an antiepileptic drug," Drug Development and Industrial Pharmacy, vol. 37, no. 3, pp. 310319, 2011.

[24] H. Meena, H. K. Pandey, M. C. Arya, and Z. Ahmed, "Shilajit: a panacea for high-altitude problems," International Journal of Ayurveda Research, vol. 1, no. 1, pp. 37-40, 2010.

[25] E. Wilson, G. V. Rajamanickam, G. P. Dubey et al., "Review on shilajit used in traditional Indian medicine," Journal of Ethnopharmacology, vol. 136, no. 1, pp. 1-9, 2011.

[26] D. Rowland, "Vitamin/mineral composition," US Patent no. $5405613,1995$.

[27] S. Ghosal, "Herbo-Mineral compositions," US Patent application no. 20030198695, 2002.

[28] S. Ghosal, "Process for preparing purified shilajit from native shilajit," US Patent no. 6440436, 2002.

[29] S. Ghosal, "Delivery system for pharmaceutical, nutritional and cosmetic ingredient," US Patent no. 6558712, 2003.

[30] R. B. Maccioni, L. Quiñones, I. Saavedra, and R. Sandoval, "Nutraceutical composition that comprises extract of shilajit, folic acid, vitamin B12 and vitamin B6 and the use thereof for preventing and/or treating neurodegenerative disease and/or the cognitive deterioration association with cerebral aging," WO 2011/041920. PCT/CL2010/000043 April, 14. 2011.

[31] R. B. Saper, R. S. Phillips, A. Sehgal et al., "Lead, mercury, and arsenic in US- and Indian-manufactured Ayurvedic medicines sold via the internet," Journal of the American Medical Association, vol. 300, no. 8, pp. 915-923, 2008.

[32] S. N. Kales and R. B. Saper, "Ayurvedic lead poisoning: an under-recognized, international problem," Indian Journal of Medical Sciences, vol. 63, no. 9, pp. 379-381, 2009.

[33] S. Singh, K. K. Mukherjee, K. D. Gill, and S. J. S. Flora, "Lead-induced peripheral neuropathy following ayurvedic medication," Indian Journal of Medical Sciences, vol. 63, no. 9, pp. 408-410, 2009. 


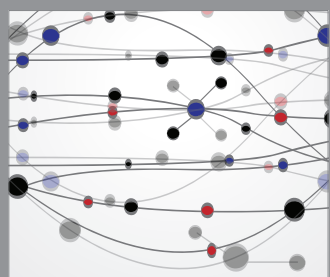

The Scientific World Journal
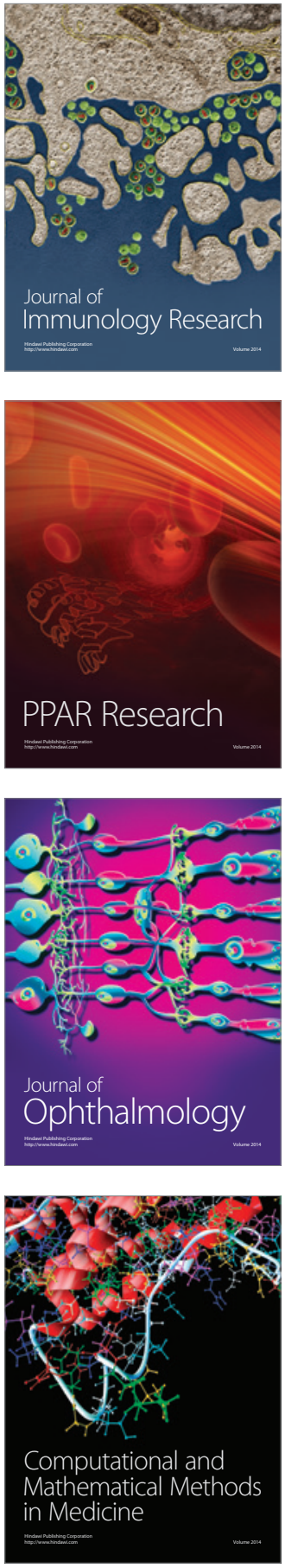

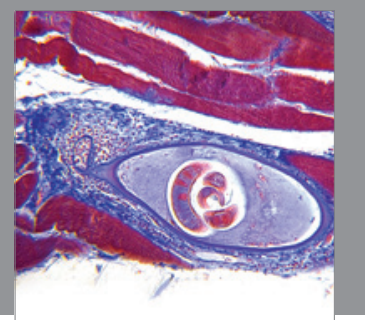

Gastroenterology

Research and Practice
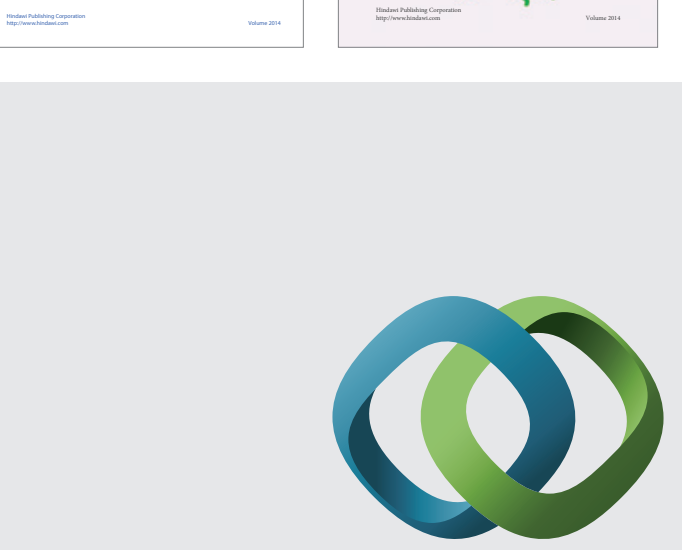

\section{Hindawi}

Submit your manuscripts at

http://www.hindawi.com
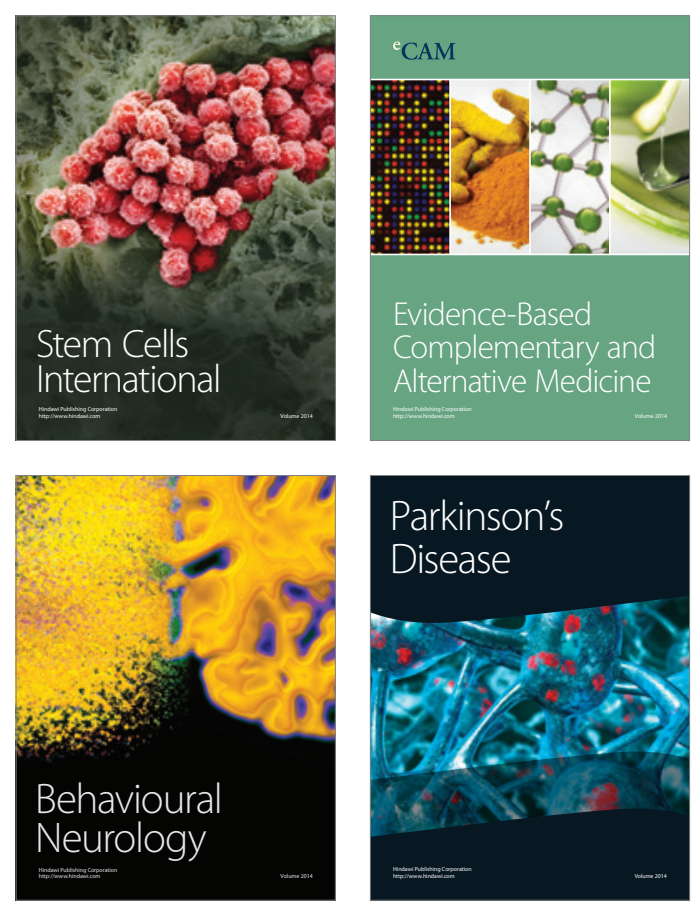

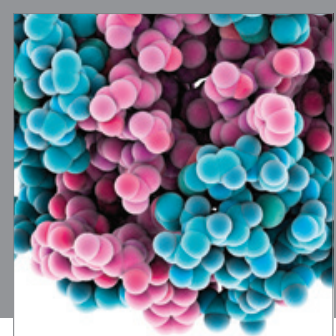

Journal of
Diabetes Research

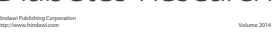

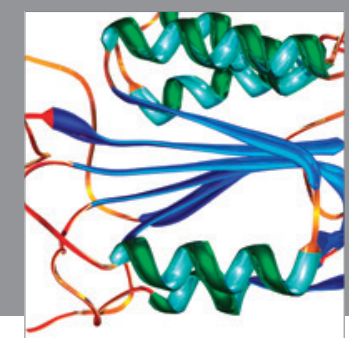

Disease Markers
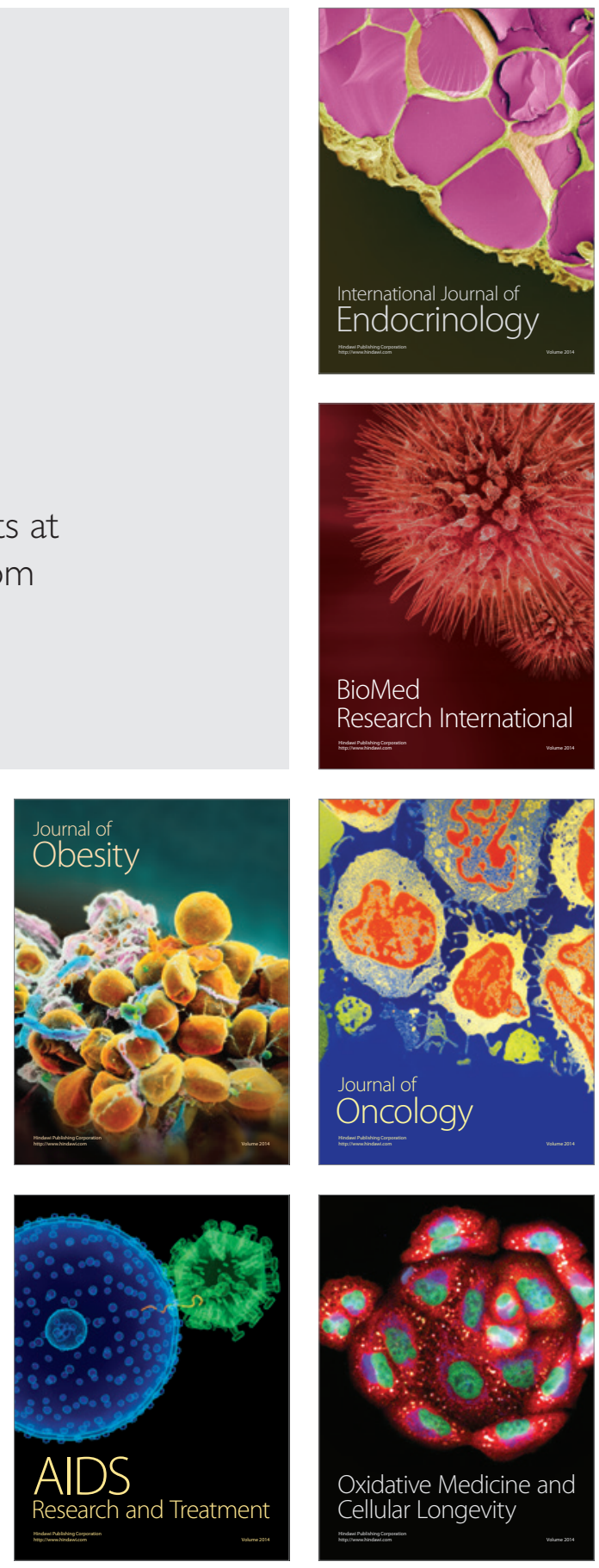